\title{
Design and Implementation of an Intelligent System for Seafarers' "Cargo stowage and securing” Assessment
}

\author{
Qingwu $^{1, \mathrm{a}}$ WANG, Weifeng $\mathrm{LI}^{1, \mathrm{~b}}$, Jian $\mathrm{ZHAO}^{1, \mathrm{c}}$ \\ ${ }^{1}$ Navigation College, Dalian Maritime University,

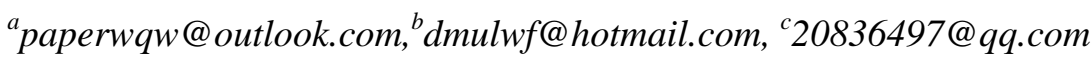 \\ supported by"the Fundamental Research Funds for the Central \\ Universities”-3132013006
}

\begin{abstract}
Cargo operation is of great importance as far as the Chief Officer's duties are concerned. "Cargo stowage and securing" is therefore one of the most important assessment courses for seafarers. The traditional assessment is however based on the subjective judgment by the assessors, which makes unfair treatment inevitably. Some seafarers even attempt to seek the unfair means in order to acquire high marks in the assessment. Such behaviors not only severely violate the fairness in light of assessment, but also eliminate the significance of evaluating the seafarers' capabilities of cargo handling and the failure of which may cause vital incidents during sea passage. As a result, intelligent system of "cargo stowage and securing" assessment for seafarers is opt to be the first priority. This paper has studied the syllabus of "cargo stowage and securing", whereupon framed a series of intelligent rules for the assessment and developed a set of intelligent assessment system based on B/S framework. The applicant is just required to answer the questions in the computer terminal and submit their answers once they have finished. This system will automatically provide the result and list down the losing marks where they failed if necessary. Applicants can make full use of this system for pre-exam practice and check the final result for the points where they fail. Thereby, the assessment result tends to be more convincing and reliable. This system has been successfully implemented for almost ten issue of assessment. The feedback from assessors appointed by MSA and students is quite optimistic. This system is deemed to be the pioneer in the field of assessment courses and classified as the first one within national domain by the experts from MSA.
\end{abstract}

Keyword: Assessment, Intelligent System, Cargo stowage and securing

\section{Introduction}

Cargo operation is one of the most significant skills as far as the seafarers' duties are concerned, which plays an important role in the operation of the ship. The 
Chief Officer, as the leader who manages the cargo, is vital in ensuring safe operation. So how to certificate the competence of the Chief Officer is the important duty of the MSA (Maritime Safety Administration).

According to the "Specification of assessment for the seafarers of P.R.C" (SPECIFATION for short), "cargo stowage and securing" is one of the five assessment courses. The traditional assessment is however based on the subjective judgment by the assessors, which makes unfair treatment inevitably. Some seafarers even attempt to seek the unfair means in order to acquire high marks in the assessment. Such behaviors not only severely violate the fairness in light of assessment, but also eliminate the significance of evaluating the seafarers' capabilities of cargo handling and the failure of which may cause vital incidents during sea passage. As a result, it is necessary to develop an assessment system without interruption of the assessor.

\section{Requirement of the Intelligent Assessment System}

General requirement. The intelligent assessment system is a kind of exam system, which consists of the following modules: basic information management, organization, monitor and analysis. The basic information module includes such function point: exam point management, exam library management, applicant management, exam sit management and parameter setup. The organization of the exam module consists of the following point: registry of the applicant and generating paper. Dispatching paper, send the start/end order, list the login status of the applicant. The function of parameter setup is to set the pass score, the exam duration etc.

The requirement from the syllabus of "cargo stowage and securing". The purpose of the assessment is to test the ability of the cargo operation for the $\mathrm{C} / \mathrm{O}$ and to check if they meet the demand of Manila Amendments to STCW Convention and Code and assessment of competition exam organized by the $\mathrm{MSA}^{[1]}$.

The assessment includes five items, which are general cargo ship stowage, bulk carrier stowage, bulk grain carrier stowage, container ship stowage and non-standard cargo securing. For C/O on ships of 3000GT or more, random one of the general cargo ship stowage and container ship stowage is compulsory, the same as random one of bulk carrier stowage, bulk grain carrier stowage and non-standard cargo securing. Please refer to the SPECIFATION for other classic $\mathrm{C} / \mathrm{O}$.

The total score of each item is 100, and the pass score is 80 . Each course item will set some “mine point”, which is critical. If the applicant can't answer them correct, they will fail the exam. 
Requirement of general cargo ship stowage. The main key points are as follows ${ }^{[2]}$ :

(1) The applicant is required to check the weight ability, volume ability and special cargo loading ability via the cargo list and load line.

(2) The max and min weight of each hold should be worked out according to the volume ratio of each hold

(3) The applicant is requested to draft the pre-stowage plan, during which the actual status of the ship should be considered, such as the isolation of dangerous cargo.

(4) The applicant is required to check that if there is something wrong with the weight and volume of each hold.

(5) This part is to evaluate the ability of the stability, trim and longitudinal strength calculation for the applicant.

(6) Tthe applicant is required to draw the formal cargo plan.

Requirement of the bulk carrier stowage. In this part the key point is as follows:

(1) The applicant is required to work out the max loading weight, according to the load line, $\mathrm{max} / \mathrm{min}$ draft and the stowage factor of the cargo.

(2) In this part, the applicant is required to allocate weight for each hold, establish the loading sequence and check the stability, trim and strength.

(3) This part is to check the ability of drawing and reading the cargo plan for the applicant.

Requirement of bulk grain stowage requirement. In this part the key point is as follows:

(1) In this part, the applicant is required to work out the max loading weight, according to the load line, max/min draft and the stowage factor of the cargo.

(2) Allocate weight for each hold and get the stability, trim and strength by the loading master

In this part, the applicant is required to allocate weight for each hold, check the stability, trim and strength.

(3) This part is to check the ability of drawing and reading the cargo plan for the applicant.

(4) The applicant is required to fill one of form of the three which are from the U.S.A, Canada and Australia.

Requirement of container ship. The main key points are as follows:

(1) Check the ability of Container Loading according to the load line, container list, loading ability of the ship, special container, etc.

(2) Compile the pre-stowage plan and get the stability, trim and strength by the loading master

In this part, the applicant is required to compile the stowage plan correctly. 
(3) In this part, the applicant is required to check the securing result properly. Requirement of securing of Non-standard cargo requirement. In this part the key point is as follows:

(1) The applicant is required to establish the securing scheme according to the ship's particular, cargo information and location of the cargo on the ship.

(2) The applicant is required to calculate the movement force banding force, overturning moment and constraining moment according to the regulation of the CCS.

\section{Implementation of the Intelligent Assessment System}

Spring, which is powerful and lightweight, is used as the framework. Hibernate is used as the database access and database mapping and Mysql is applied. Database connection pool is based on Proxool ${ }^{[3-5]}$.

Implementation of the general cargo stowage module. The method of creating control dynamically is used to resolve the problem of allocating the cargo. Each hold can be divided into many parts for different cargoes. A tab control is used by each hold. There is one tabpage in the tab control for default and one more tabpage is added for one more layer.

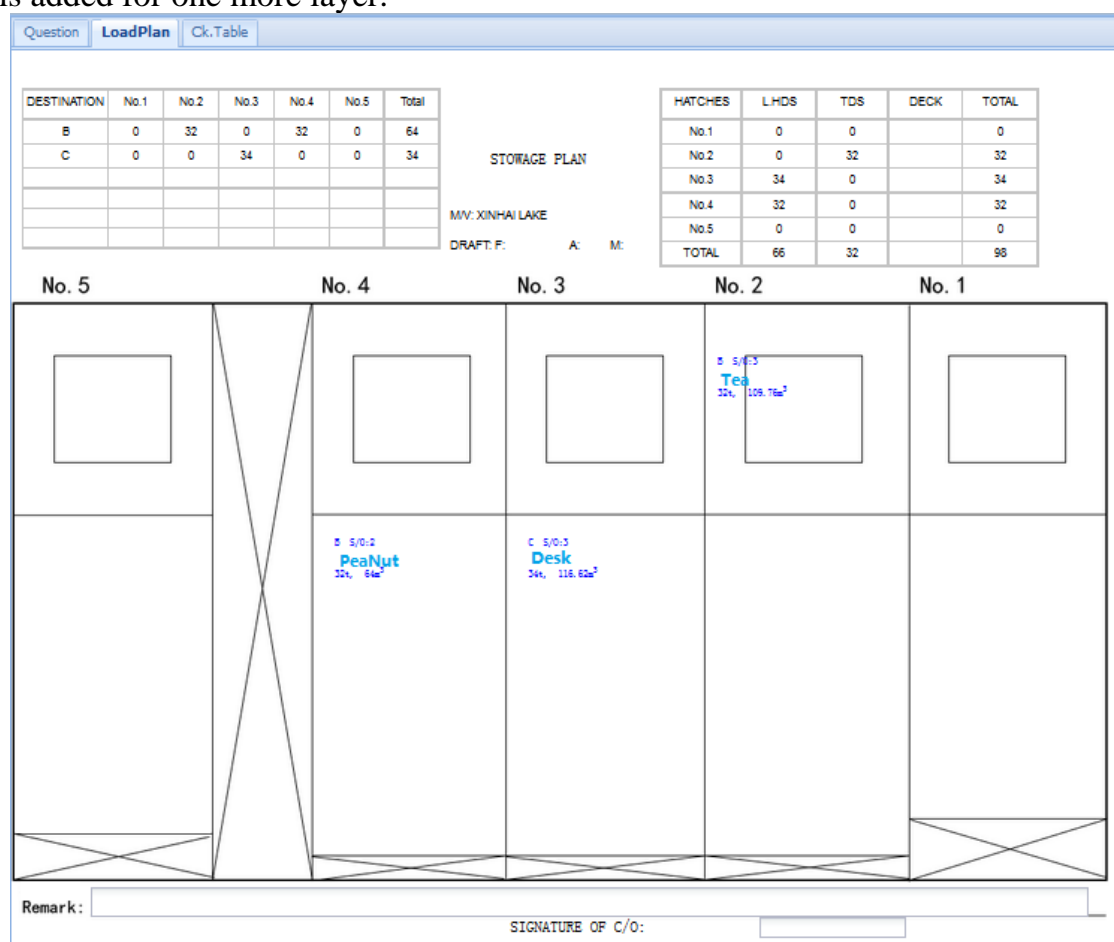

Fig. 1. Final interface of general cargo ship stowage module 
Tab. 1. Details of class GerCargoPos

\begin{tabular}{ccl}
\hline Member & DataType & Remark \\
\hline ID & String & primary key \\
TANK & Integer & Id. of hold \\
TIER & Integer & 1:bottem,2: tween deck \\
SO & String & Shipping Order \\
cargoId & Integer & Foreign key \\
W & Double & Weight of cargo \\
V & Double & Volume of cargo \\
DP & String & Destination Port \\
idx & Integer & Transversal index \\
pLayout & Integer & Longitudinal index \\
layer & Integer & Layer num. \\
\hline & &
\end{tabular}

A class named GerCargoPos is defined for each part, the details of which are shown in tab. 1.

The final interface of general cargo ship stowage module is shown in fig. 1.

Implementation of the bulk carrier module. A capsized carrier with nine holds and handy with five holds are used in the module. The stable and strength information can be figured out by the loading master. After the loading plan is confirmed, the loading sequence will get its turn. All of the stable and strength information should meet the requirement. Fig. 2 shows the final interface.

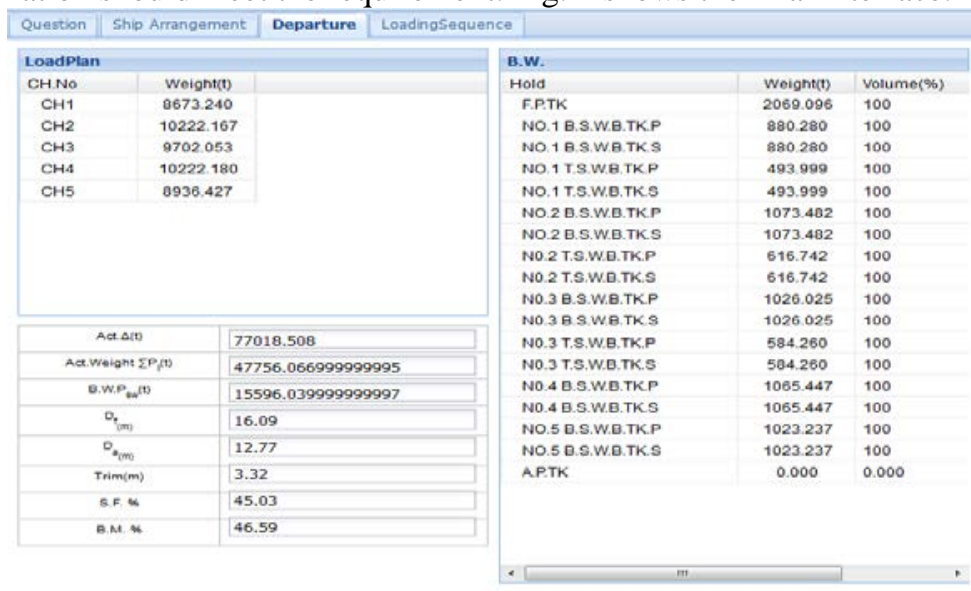

Fig. 2. Final interface of bulk carrier stowage module 


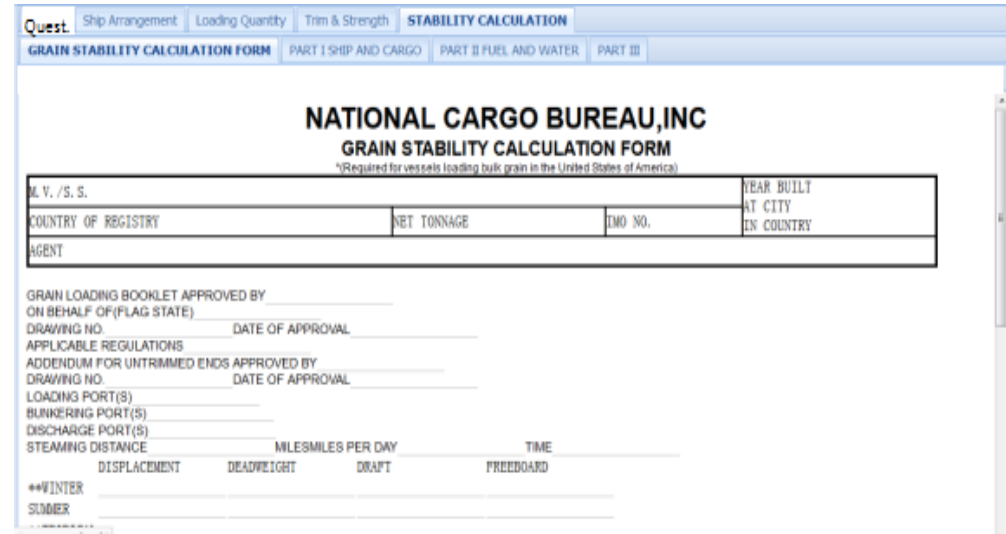

Fig. 3. Final interface of bulk grain carrier stowage module

Implementation of the bulk grain carrier module. There are four windows in this module, which are ship arrangement, loading quantity, strength \& trim and the grain stability calculation form. The former three windows are similar to the bulk carrier module. So the last window is of the most significant. The relevant from is used as the background of the last windows, and some textbox is added in the blanks. Fig 3 shows the form of U.S.A.

Implementation of container ship stowage module. The emphases of this module are the allocating window seeing fig. 4. In this window, one button is used for one container. If the button is clicked, the position of the container will be shown automatically.

Isolation of dangerous container is another key point. To check the loading plan meets the requirement or not, the number of containers between two can be figured out with the formula (1).

$$
\mathrm{C}=\left|(-1)^{r 1} *\left[\frac{r 1}{2}\right]-(-1)^{r 2} *\left[\frac{r 2}{2}\right]\right|
$$

In the formula, r1 and r2 is the column number, $01 / 03 / 05 \ldots .$. to starboard, $02 / 04 / 06 \ldots$...to port, 00 in the middle. Whether the 00 column is exist or not depends on the ship. If the 00 column exist, the result should be added by 1 . $C=0$ means the two container are in the same column. Symbol [] stands for the ceiling integer. 


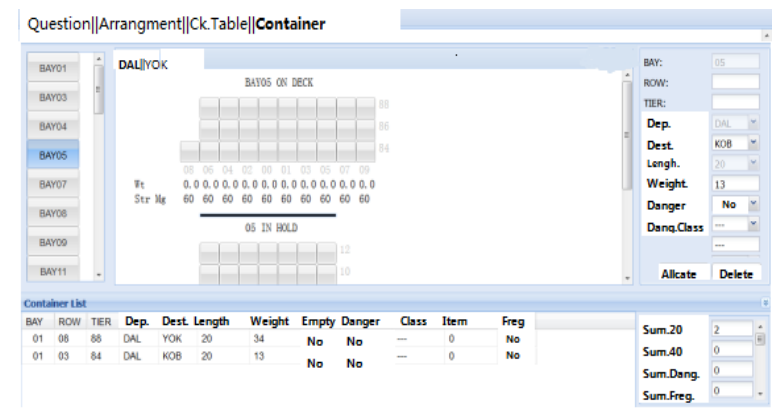

Fig 4. Final interface of container ship stowage module

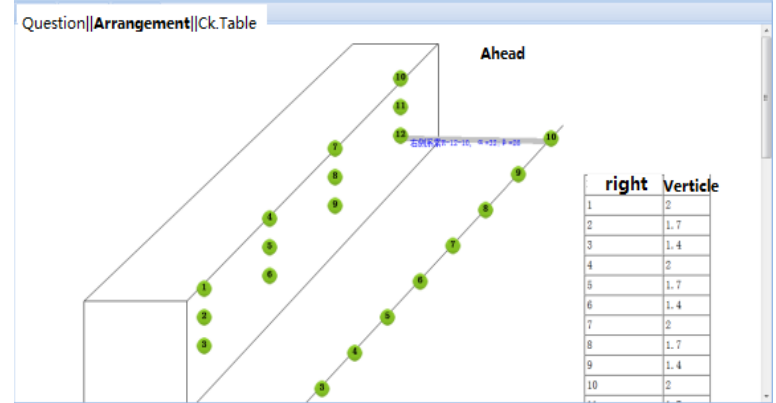

Fig 5. Final interface of non-standard

cargo securing module

Implementation of the non-standard cargo module. This module is divided into two parts which are establishment and check of the securing scheme. Fig 5 is the securing scheme establishment. After the two banding point on the cargo and the body of the ship are defined, the banding line connecting the two banding point will be created automatically. At the same time, the angle between the line and the ship will be figured out. The other window consists of textbox, which makes it simple to be implemented.

\section{Conclusion}

This paper introduces the requirement, design and implementation of the "cargo stowage and securing” intelligent assessment system. The system had been used for ten times in the Liaoning MSA. The result shows that the system is of reasonable design and convenient for application. This system is deemed to be the pioneer in the field of assessment courses and classified as the first one within national domain by the experts from MSA.

\section{Reference:}

[1]M.S.A. China. Specification of assessment for the seafarers of P.R.C[M].Dalian: Dalian Maritime Express.2012. 
[2]Baijun TIAN. Ship’s Structure and Cargo operation[M]. Dalian: Dalian Maritime Express.2013.

[3]Zhongguo MA. Cargo stowage and lashing Assessment - Examination System Design and Implementation[D]. Dalian:Dalian Maritime University.2012.

[4]Ext JS in Action [M]. Manning Publications.2014.

[5]Why MySQL[EB/OL].http://www.mysql.com/why-mysql. 